\title{
Substâncias psicoativas e saúde mental em profissionais de enfermagem da Estratégia Saúde da Família
}

Vanessa Cristina Bertussi ${ }^{1}$, Marcelle Aparecida de Barros Junqueira ${ }^{2}$, Carla Denari Giuliani ${ }^{3}$, Rubianne Monteiro Calçado ${ }^{4}$, Frank José Silveira Miranda ${ }^{5}$, Manoel Antônio dos Santos ${ }^{6}$, Sandra Cristina Pillon ${ }^{7}$

\section{RESUMO}

Este estudo teve por objetivo conhecer a associação entre uso de substâncias psicoativas e depressão, estresse e ansiedade entre profissionais de enfermagem de equipes da Estratégia Saúde da Família. Estudo transversal realizado com 112 participantes. Foram utilizados instrumentos de rastreamento para uso abusivo de álcool e outras drogas e uma escala que avalia sintomas de depressão, estresse e ansiedade. Os resultados mostraram que $44,6 \%$ dos profissionais consumiam álcool no padrão binge, a maioria do sexo masculino, 16,2\% apresentavam sintomas de depressão, $15,2 \%$ de estresse e $23,2 \%$ de ansiedade. 0 uso de álcool em binge foi associado à depressão $(p=, 035)$. Foram encontradas correlações positivas entre depressão, estresse e ansiedade e correlações negativas entre estresse, idade e tempo de trabalho. $O$ uso problemático de álcool e os sintomas de transtornos mentais estão correlacionados e precisam ser melhor investigados entre os profissionais de enfermagem da Estratégia Saúde da Família.

Descritores: Profissionais de Enfermagem; Saúde do Trabalhador; Atenção Primária à Saúde.

\footnotetext{
${ }^{1}$ Enfermeira, Mestre em Saúde Ambiental e Saúde do Trabalhador. Apoiadora Institucional de Setor Sanitário da Prefeitura Municipal de Uberlândia. Uberlândia, MG, Brasil. E-mail: vbertussi@yahoo.com.br.

2 Enfermeira, Doutora em Enfermagem Psiquiátrica. Professora Adjunta da Universidade Federal de Uberlândia. Uberlândia, MG, Brasil. E-mail: marcellebarros@ufu.br.

${ }^{3}$ Enfermeira, Doutora em História. Professora Adjunta da Universidade Federal de Uberlândia. Uberlândia, MG, Brasil. E-mail: denarigiuliani@bol.com.br.

${ }^{4}$ Acadêmica do curso de Graduação em Enfermagem pela Universidade Federal de Uberlândia. Uberlândia, MG, Brasil. E-mail: rubiannemonteiro@hotmail.com.

${ }^{5}$ Enfermeiro, Doutor em Ciências Médicas. Professor Adjunto da Universidade Federal de Uberlândia. Uberlândia, MG, Brasil. E-mail: frankenfermeiro@yahoo.com.br.

${ }_{6}$ Psicólogo, Doutor em Psicologia Clínica. Professor Doutor da Faculdade de Filosofia Ciências e Letras de Ribeirão Preto, da Universidade de São Paulo. Ribeirão Preto, SP, Brasil. E-mail: manoelmasantos@gmail.com.

7 Enfermeira, Doutora em Psiquiatria e Psicologia Médica. Professora Titular da Escola de Enfermagem de Ribeirão Preto da Universidade de São Paulo. Ribeirão Preto, SP, Brasil. E-mail: pillon@eerp.usp.br.
}

Artigo recebido: 12/07/2017. Artigo aprovado: 13/03/2018. Artigo publicado: 03/10/2018.

\section{Como citar esse artigo:}

Bertussi VC, Junqueira MAB, Giuliani CD, Calçado RM, Miranda FJS, Santos MA, et al. Substâncias psicoativas e saúde mental em profissionais de enfermagem da Estratégia Saúde da Família. Rev. Eletr. Enf. [Internet]. 2018 [acesso em: ];20:v20a21. Disponível em: https://doi.org/10.5216/ree.v20.47820. 


\section{INTRODUÇÃO}

A Estratégia Saúde da Família (ESF) é o modelo prioritário da Atenção Primária à Saúde (APS), que no Brasil também é conhecida como Atenção Básica ( $A B)$. Esse modelo preconiza um conjunto de ações de caráter individual e coletivo, situadas no primeiro nível de atenção dos sistemas de saúde, voltadas à promoção de saúde, prevenção de agravos, tratamento e reabilitação(1).

$O$ trabalho desenvolvido pelos profissionais de enfermagem influencia diretamente a qualidade da atenção em saúde prestada aos pacientes e comunidade. Porém, os trabalhadores envolvidos na ESF se deparam com diversas barreiras em seu cotidiano, como escassez de recursos humanos, excesso de demanda, jornada de trabalho longa e exaustiva, sobrecarga resultante das demandas administrativas, insatisfação salarial e déficits na estrutura física dos equipamentos de saúde. Esses problemas, aliados a falhas no funcionamento da rede de atenção do Sistema Único de Saúde (SUS) e à desarticulação entre os diversos serviços que compõem a rede intersetorial resultam em sobrecarga e podem incrementar a insatisfação com o trabalho, desencadeando sofrimento(2).

Os trabalhadores da ESF apresentam elevadas prevalências de problemas de saúde, inclusive de saúde mental, tais como depressão, ansiedade, suscetibilidade ao estresse, insônia, fadiga, irritabilidade, dificuldade de concentração, alterações de memória e queixas somáticas ${ }^{(3)}$. Ademais, evidências apontam que depressão, ansiedade, estresse e cansaço crônico podem influenciar o uso e abuso de substâncias psicoativas, como álcool, tabaco, tranquilizantes e outras, uma vez que são manifestações de transtornos mentais comumente relacionados ao adoecimento associado ao trabalho ${ }^{(4-7)}$.

A pesquisa se justifica à medida que se propõe a avaliar as associações entre o uso de drogas e a presença de sintomas de transtornos mentais em profissionais de enfermagem que atuam nas equipes da ESF, podendo colaborar para a identificação de perfis epidemiológicos e possível deteç̧ão precoce de problemas relacionados a esses agravos. Na literatura brasileira e estrangeira há um número incipiente de estudos que se dedicam a investigar essas associações. Considerando o exposto, este estudo teve por objetivo conhecer a associação entre uso de substâncias psicoativas e depressão, estresse e ansiedade entre profissionais de enfermagem de equipes da ESF.

\section{MATERIAIS E MÉTODOS}

Estudo transversal de abordagem quantitativa. A pesquisa foi desenvolvida com profissionais de enfermagem das equipes do ESF de um município do interior do Estado de Minas Gerais, Brasil. A coleta de dados foi realizada entre os meses de maio a agosto de 2016.

Nesse período o município contava com 74 equipes de ESF, compostas por 170 profissionais, sendo 74 enfermeiros e 96 auxiliares e técnicos de enfermagem. Mediante o cálculo amostral do tipo aleatório simples foi estimado um total de 103 participantes, considerando um intervalo de confiança [IC] de 95\% e erro amostral de $6 \%$. Todavia todos os profissionais de enfermagem foram convidados a participar do estudo. Assim, a amostra foi composta por 112 profissionais que concordaram em participar da pesquisa.

O estudo foi aprovado pelo Comitê de Ética em Pesquisa da Universidade Federal de Uberlândia (CEP/UFU), sob parecer $n$ ㅇ 1.585.311. Realizou-se um contato prévio com os responsáveis pelas unidades e foram agendados 
os horários para a realização da coleta de dados de modo a não interferir com a rotina de trabalho. Durante as visitas aos locais todos os profissionais de enfermagem que aceitaram participar do estudo assinaram o Termo de Consentimento Livre e Esclarecido (TCLE). Em seguida, foi entregue a cada participante uma via impressa do instrumento de coleta de dados autoaplicável, composto por informações sociodemográficas e ocupacionais, e três instrumentos padronizados:

a) Alcohol, Smoking and Substance Involvement Screening Test (ASSIST): instrumento de triagem do uso de álcool, tabaco e outras substâncias ${ }^{(8)}$;

b) Alcohol Use Disorder Identification Test-Consumption (AUDIT-C): instrumento de rastreamento do uso de álcool que avalia a quantidade e frequência do uso e o beber no padrão binge (definido como cinco ou mais doses em uma única ocasião para homens e quatro doses ou mais para mulheres) ${ }^{(9)}$;

c) Depression, Anxiety and Stress Scale (DASS-21): instrumento que avalia os sintomas de depressão, ansiedade e estresse ${ }^{(10)}$.

Todos os instrumentos aplicados foram validados para o contexto brasileiro e apresentaram propriedades psicométricas adequadas, com bons níveis de confiabilidade interna.

Os dados obtidos foram submetidos a análises estatísticas descritivas e bivariadas. Os testes estatísticos empregados foram o Qui-Quadrado $\left(\chi^{2}\right)$ para avaliar a associação das variáveis categóricas e o Coeficiente de Correlação de Spearman (Ro) para verificar a correlação entre duas amostras dependentes. Para todas as análises o nível de significância (valor de $p$ ) adotado foi 0,05. Os resultados foram expressos em frequência absoluta (n) e relativa (\%).

\section{RESULTADOS}

A amostra foi composta por 112 profissionais de enfermagem, caracterizados predominantemente pelo sexo feminino, com média de idade de 37,4 anos, que trabalhavam como técnicos de enfermagem e enfermeiros, com tempo médio de atuação profissional de 9, 12 anos.

Tabela 1: Caracterização sociodemográfica dos profissionais de enfermagem de equipes da ESF ( $N=112)$. Uberlândia, MG, Brasil, 2016.

\begin{tabular}{cccc}
\hline & & $\mathbf{N}$ & \% \\
\hline \multirow{2}{*}{ Sexo } & Feminino & 105 & 93,8 \\
& Masculino & 7 & 6,2 \\
Estado civil & Casado & 74 & 66,1 \\
& Solteiro & 33 & 29,5 \\
& Viúvo & 4 & 3,6 \\
Cargo & Não respondeu & 1 & 0,9 \\
& Auxiliar de Enfermagem & 5 & 4,5 \\
Escolaridade & Técnico em Enfermagem & 54 & 48,2 \\
& Enfermeiro & 53 & 47,3 \\
& Ensino Médio/Fundamental & 43 & 44,4 \\
& Ensino Superior & 54 & 48,2 \\
\hline
\end{tabular}

Observa-se que mais da metade dos participantes já fez uso de álcool uma vez na vida e quase a metade relatou já ter consumido bebida alcoólica no padrão binge ${ }^{(9)}$. O consumo de álcool pelo menos uma vez na vida 
Bertussi VC, Junqueira MAB, Giuliani CD, Calçado RM, Miranda FJS, Santos MA, et al.

foi maior junto aos profissionais com ensino superior e o consumo no padrão binge foi mais expressivo junto aos participantes do sexo masculino, com diferenças estatisticamente significantes (Tabela 2).

Tabela 2: Padrões de consumo de álcool dos profissionais de enfermagem da ESF (N=112). Uberlândia, MG, Brasil, 2016.

\begin{tabular}{|c|c|c|c|c|c|c|c|}
\hline & & \multicolumn{2}{|c|}{ Uso na vida } & \multicolumn{2}{|c|}{$\begin{array}{c}\text { Uso nos últimos três } \\
\text { meses }\end{array}$} & \multicolumn{2}{|c|}{ Uso em binge } \\
\hline & & $\mathbf{N}$ & $\%$ & $\mathbf{N}$ & $\%$ & $\mathbf{N}$ & $\%$ \\
\hline & TOTAL & 58 & 51,8 & 44 & 39,3 & 55 & 44,6 \\
\hline \multirow{4}{*}{ Sexo } & Feminino & 55 & 54,5 & 41 & 43,2 & 44 & 41,9 \\
\hline & Masculino & 3 & 60,0 & 3 & 75,0 & 06 & 85,7 \\
\hline & & \multicolumn{2}{|c|}{$\chi^{2}(1)=, 059$} & \multicolumn{2}{|c|}{$\chi^{2}(1)=1,576$} & \multicolumn{2}{|c|}{$\chi^{2}(1)=5,097$} \\
\hline & & \multicolumn{2}{|c|}{$p=, 808$} & \multicolumn{2}{|c|}{$p=, 209$} & \multicolumn{2}{|c|}{$p=, 024^{*}$} \\
\hline \multirow{5}{*}{ Cargo } & Auxiliar de Enfermagem & 4 & 40 & 4 & 40 & 6 & 50 \\
\hline & Técnico em Enfermagem & 20 & 47,6 & 13 & 35,1 & 19 & 43,2 \\
\hline & Enfermeiro & 33 & 64,7 & 25 & 51 & 24 & 45,3 \\
\hline & & \multicolumn{2}{|c|}{$\chi^{2}(2)=3,775$} & \multicolumn{2}{|c|}{$\chi^{2}(2)=2,225$} & \multicolumn{2}{|c|}{$\chi^{2}(2)=, 182$} \\
\hline & & \multicolumn{2}{|c|}{$p=, 151$} & \multicolumn{2}{|c|}{$p=, 329$} & \multicolumn{2}{|c|}{$p=, 913$} \\
\hline \multirow{4}{*}{ Companhei-ro(a) } & Sim & 38 & 54,3 & 13 & 20,6 & 29 & 39,2 \\
\hline & Não & 20 & 57,1 & 6 & 17,1 & 20 & 51,4 \\
\hline & & \multicolumn{2}{|c|}{$\chi^{2}(1)=, 077$} & \multicolumn{2}{|c|}{$\chi^{2}(1)=, 176$} & \multicolumn{2}{|c|}{$\chi^{2}(1)=2,211$} \\
\hline & & \multicolumn{2}{|c|}{$p=, 781$} & \multicolumn{2}{|c|}{$p=, 675$} & \multicolumn{2}{|c|}{$p=, 137$} \\
\hline \multirow{4}{*}{ Escolaridade } & Ensino Médio/Fundamental & 19 & 52,8 & 15 & 46,9 & 18 & 47,4 \\
\hline & Ensino Superior & 35 & 67,3 & 26 & 53,1 & 27 & 50 \\
\hline & & \multicolumn{2}{|c|}{$\chi^{2}(2)=6,389$} & \multicolumn{2}{|c|}{$\chi^{2}(2)=4,208$} & \multicolumn{2}{|c|}{$\chi^{2}(2)=1,652$} \\
\hline & & \multicolumn{2}{|c|}{$p=, 041^{*}$} & \multicolumn{2}{|c|}{$p=, 122$} & \multicolumn{2}{|c|}{$p=, 438$} \\
\hline
\end{tabular}

Nota: Teste de Qui-quadrado $\left(\chi^{2}\right)$, *valor de $\mathrm{p}<0,05$.

Quanto ao consumo de outras substâncias (exceto álcool), de modo geral percebe-se um uso relativamente baixo referido nos últimos três meses ou que pudesse ser considerado abusivo entre os profissionais de enfermagem da ESF. O uso ao menos uma vez na vida e o uso abusivo foram predominantes entre os profissionais do sexo masculino, com diferenças estatisticamente significantes (Tabela 3).

Tabela 3: Uso de substâncias psicoativas (exceto álcool) entre profissionais de ESF (N=112). Uberlândia, MG, Brasil, 2016.

\begin{tabular}{|c|c|c|c|c|c|c|c|}
\hline & & \multicolumn{2}{|c|}{ Uso na vida } & \multicolumn{2}{|c|}{$\begin{array}{l}\text { Uso nos últimos } \\
\text { três meses }\end{array}$} & \multicolumn{2}{|c|}{ Uso abusivo } \\
\hline & & $\mathbf{N}$ & $\%$ & $\mathbf{N}$ & $\%$ & $\mathbf{N}$ & $\%$ \\
\hline \multicolumn{2}{|r|}{ TOTAL } & 12 & 10,7 & 4 & 3,6 & 4 & 3,6 \\
\hline \multirow{4}{*}{ Sexo } & Feminino & 10 & 11,9 & 3 & 3,5 & 3 & 4,2 \\
\hline & Masculino & 2 & 100 & 1 & 20 & 1 & 50 \\
\hline & & \multicolumn{2}{|c|}{$\chi^{2}(1)=12,627$} & \multicolumn{2}{|c|}{$\chi^{2}(1)=3,016$} & \multicolumn{2}{|c|}{$\chi^{2}(1)=5,097$} \\
\hline & & \multicolumn{2}{|c|}{$p<, 001$} & \multicolumn{2}{|c|}{$p=, 082$} & \multicolumn{2}{|c|}{$p=, 024^{*}$} \\
\hline \multirow{6}{*}{ Cargo } & Auxiliar de Enfermagem & 1 & 12,5 & - & - & - & - \\
\hline & Técnico em Enfermagem & 2 & 6,2 & 2 & 6,1 & 2 & 8 \\
\hline & Enfermeiro & 9 & 20,5 & 2 & 4,4 & 2 & 5 \\
\hline & & \multicolumn{2}{|c|}{$\chi^{2}(2)=3,076$} & \multicolumn{2}{|c|}{$\chi^{2}(2)=, 652$} & \multicolumn{2}{|c|}{$\chi^{2}(2)=, 720$} \\
\hline & & \multicolumn{2}{|c|}{$p=, 215$} & \multicolumn{2}{|c|}{$p=, 722$} & \multicolumn{2}{|c|}{$p=, 698$} \\
\hline & Sim & 7 & 11 & 2 & 3,4 & 2 & 4,1 \\
\hline \multirow{4}{*}{$\begin{array}{l}\text { Vive com companheiro } \\
\text { (a) }\end{array}$} & Não & 5 & 22,7 & 2 & 7,4 & 2 & 10 \\
\hline & & \multicolumn{2}{|c|}{$\chi^{2}(2)=2,135$} & \multicolumn{2}{|c|}{$\chi^{2}(2)=0,843$} & \multicolumn{2}{|c|}{$\chi^{2}(2)=1,132$} \\
\hline & & \multicolumn{2}{|c|}{$p=, 344$} & \multicolumn{2}{|c|}{$p=, 656$} & \multicolumn{2}{|c|}{$p=, 568$} \\
\hline & Ensino Médio/Fundamental & 3 & 12 & 2 & 7,1 & - & - \\
\hline \multirow{3}{*}{ Escolaridade } & Ensino Superior & 9 & 20,5 & 2 & 4,3 & 12 & 14,4 \\
\hline & & \multicolumn{2}{|c|}{$\chi^{2}(2)=1,447$} & \multicolumn{2}{|c|}{$\chi^{2}(2)=, 387$} & \multicolumn{2}{|c|}{$\chi^{2}(2)=, 504$} \\
\hline & & \multicolumn{2}{|c|}{$p=, 485$} & \multicolumn{2}{|c|}{$p=, 824$} & \multicolumn{2}{|c|}{$p=, 478$} \\
\hline
\end{tabular}


Em relação aos sintomas sugestivos de depressão, ansiedade e estresse (DASS), os resultados apontaram prevalência de sintomas de ansiedade, seguidos de depressão e estresse, no entanto, sem diferenças estatisticamente significantes quando consideradas as características sociodemográficas (Tabela 4).

Tabela 4: Características sociodemográficas e sintomas de depressão, estresse e ansiedade entre profissionais de ESF (N=112). Uberlândia, MG, Brasil, 2016.

\begin{tabular}{|c|c|c|c|c|c|c|c|}
\hline & & \multicolumn{6}{|c|}{ Sintomas } \\
\hline & & \multicolumn{2}{|c|}{ Depressão } & \multicolumn{2}{|c|}{ Estresse } & \multicolumn{2}{|c|}{ Ansiedade } \\
\hline & & $\mathbf{N}$ & $\%$ & $\mathbf{N}$ & $\%$ & $\mathbf{N}$ & $\%$ \\
\hline \multicolumn{2}{|r|}{ TOTAL } & 18 & 16,1 & 17 & 15,2 & 26 & 23,2 \\
\hline & Feminino & 17 & 18,7 & 17 & 18,9 & 25 & 26,9 \\
\hline & Masculino & 1 & 16,7 & - & - & 1 & 16,7 \\
\hline \multirow[t]{4}{*}{ Sexo } & & \multicolumn{2}{|c|}{$\chi^{2}(1)=, 015$} & \multicolumn{2}{|c|}{$\chi^{2}(1)=1,377$} & \multicolumn{2}{|c|}{$\chi^{2}(1)=, 304$} \\
\hline & & \multicolumn{2}{|c|}{$p<, 902$} & \multicolumn{2}{|c|}{$p=, 241$} & \multicolumn{2}{|c|}{$p=, 584$} \\
\hline & Auxiliar de Enfermagem & 1 & 10 & - & & - & - \\
\hline & Técnico em Enfermagem & 9 & 25,7 & 8 & 25,3 & 12 & 32,4 \\
\hline \multirow[t]{5}{*}{ Cargo } & Enfermeiro & 8 & 16 & 9 & 18 & 14 & 25 \\
\hline & & \multicolumn{2}{|c|}{$\chi^{2}(2)=1,848$} & \multicolumn{2}{|c|}{$\chi^{2}(2)=2,888$} & \multicolumn{2}{|c|}{$\chi^{2}(2)=4,296$} \\
\hline & & \multicolumn{2}{|c|}{$p=, 397$} & \multicolumn{2}{|c|}{$p=, 236$} & \multicolumn{2}{|c|}{$p=, 117$} \\
\hline & Sim & 12 & 19 & 10 & 16,1 & 16 & 25 \\
\hline & Não & 06 & 18,2 & 07 & 20,6 & 10 & 29,4 \\
\hline \multicolumn{8}{|c|}{ Vive com companheiro } \\
\hline & & \multicolumn{2}{|c|}{$\chi^{2}(1)=, 011$} & \multicolumn{2}{|c|}{$\chi^{2}(1)=, 300$} & \multicolumn{2}{|c|}{$\chi^{2}(1)=, 222$} \\
\hline & & \multicolumn{2}{|c|}{$p=, 918$} & \multicolumn{2}{|c|}{$p=, 584$} & \multicolumn{2}{|c|}{$p=, 638$} \\
\hline \multirow{4}{*}{ Escolaridade } & Ensino Médio/Fundamental & 07 & 21,9 & 06 & 18,8 & 10 & 27,5 \\
\hline & Ensino Superior & 08 & 15,7 & 09 & 17,6 & 14 & 28,2 \\
\hline & & \multicolumn{2}{|c|}{$\chi^{2}(1)=, 509$} & \multicolumn{2}{|c|}{$\chi^{2}(1)=, 016$} & \multicolumn{2}{|c|}{$\chi^{2}(1)=, 039$} \\
\hline & & \multicolumn{2}{|c|}{$p=, 476$} & \multicolumn{2}{|c|}{$p=, 899$} & \multicolumn{2}{|c|}{$p=, 844$} \\
\hline
\end{tabular}

Nota: Teste de Qui-quadrado $\left(\chi^{2}\right)$, *valor de $p<0,05$.

Quanto à associação entre uso de álcool, sintomas de depressão, estresse e ansiedade, pôde-se observar que mais de um quarto dos profissionais com consumo de álcool em padrão binge apresentavam sintomas de depressão, com diferenças estatisticamente significantes (Tabela 5).

Tabela 5: Uso de álcool no padrão binge, outras drogas e sintomas de depressão, estresse e ansiedade entre profissionais de ESF ( $\mathrm{N}=112$ ). Uberlândia, MG, Brasil, 2016.

\begin{tabular}{|c|c|c|c|c|}
\hline & \multicolumn{2}{|c|}{ Uso de álcool em binge } & \multicolumn{2}{|c|}{ Uso abusivo de drogas (exceto álcool) } \\
\hline & $\mathbf{N}$ & $\%$ & $\mathbf{N}$ & $\%$ \\
\hline & 12 & 27,9 & 1 & 25 \\
\hline \multirow[t]{3}{*}{ Depressão } & \multicolumn{2}{|c|}{$\chi^{2}(1)=4,468$} & \multicolumn{2}{|c|}{$\chi^{2}(1)=, 105$} \\
\hline & \multicolumn{2}{|c|}{$p=, 035^{*}$} & \multicolumn{2}{|c|}{$p=, 745$} \\
\hline & 8 & 19 & 1 & 25 \\
\hline \multirow[t]{3}{*}{ Estresse } & \multicolumn{2}{|c|}{$\chi^{2}(1)=, 092$} & \multicolumn{2}{|c|}{$\chi^{2}(1)=, 043$} \\
\hline & \multicolumn{2}{|c|}{$p=, 762$} & \multicolumn{2}{|c|}{$p=, 835$} \\
\hline & 13 & 31 & 1 & 25 \\
\hline \multirow[t]{2}{*}{ Ansiedade } & \multicolumn{2}{|c|}{$\chi^{2}(2)=, 828$} & \multicolumn{2}{|c|}{$\chi^{2}(1)=, 003$} \\
\hline & \multicolumn{2}{|c|}{$p=, 363$} & \multicolumn{2}{|c|}{$p=, 959$} \\
\hline
\end{tabular}

Nota: Teste de Qui-quadrado $\left(\chi^{2}\right)$, *valor de $p<0,05$.

$\mathrm{Na}$ análise de correlação, utilizando o Teste de Correlação por Postos de Spearman, foi observada correlação positiva entre depressão e uso de álcool ( $r=, 227 p=0,047)$ (dados não apresentados em tabela). Correlações fortes foram detectadas entre depressão e estresse $(r=, 635 p<0,001)$, depressão e ansiedade $(r=$ 
,785 $p<0,001)$, bem como entre estresse e ansiedade $(r=, 785 p<0,001)$. Por fim, observaram-se correlações negativas entre estresse e idade $(r=-, 268 p=0,021)$.

\section{DISCUSSÃO}

Em relação às características sociodemográficas observou-se predominância do sexo feminino, corroborando resultados de estudos que apontam à feminização do trabalho em diversas área da saúde ${ }^{(3)}$. A idade dos participantes deste estudo se aproximou do observado por estudo anterior desenvolvido junto a equipes de Saúde da Família do Sul e do Nordeste do Brasil, que apresentaram média de idade de 37,8 anos ${ }^{(3)}$.

No que concerne ao uso de substâncias psicoativas, um dado preocupante revelado por esta pesquisa refere-se à frequência de binge drinking observada entre os participantes, semelhante ao que foi encontrado em um estudo realizado na Colômbia, que mostrou que $60 \%$ dos profissionais consumiram bebida alcoólica nos últimos três meses, sendo que em 8,3\% foi identificado uso abusivo ou dependência de álcool|(11).

Pesquisas que investigaram o nível de consumo de álcool no padrão binge por profissionais de enfermagem apresentam índices semelhantes aos encontrados na população geral ${ }^{(12-13)}$. O uso de álcool em binge pode estar associado não apenas aos transtornos mentais, mas também a problemas físicos e sociais ${ }^{(14)}$.

Outro resultado do presente estudo refere-se ao consumo de outras substâncias (exceto o álcool) entre profissionais da ESF, com o predomínio do sexo masculino em uso na vida e consumo abusivo entre os trabalhadores do sexo masculino. Esse resultado também foi observado em outros estudos ${ }^{(12,15)}$ que investigaram o uso de substâncias (exceto álcool). Estudo realizado no Canadá identificou o uso abusivo de drogas (exceto álcool) ente $1,9 \%$ dos participantes ${ }^{(15)}$. Na Colômbia, um inquérito entre enfermeiras que atuavam na $A B$ mostrou que $45 \%$ haviam feito uso de tabaco, $40 \%$ de energéticos e $20 \%$ de antidepressivos e barbitúricos ${ }^{(11)}$.

Um complicador importante para os profissionais de enfermagem que fazem uso abusivo de álcool ou de outras drogas é o fato de enfrentarem sentimentos de estigmatização, culpa e vergonha, o que acaba dificultando ou retardando a busca precoce de ajuda especializada. Além disso, o uso de psicotrópicos está muitas vezes associado a fatores intrínsecos (dificuldades para lidar com a morte, dor e sofrimento que permeiam o cotidiano laboral) e extrínsecos ao exercício profissional (carga horária excessiva, infraestrutura inadequada, acúmulo de tarefas, excesso de responsabilidades) ${ }^{(12)}$.

O abuso de substâncias entre profissionais de enfermagem apresenta dois desdobramentos negativos: um relacionado à própria saúde do profissional e o outro que diz respeito ao vínculo mantido com os pacientes. Dessa forma, além de contribuir para aumentar o volume de afastamentos e reivindicações de aposentadoria por incapacitação e invalidez, o abuso de substâncias pode estar relacionado ao aumento no número de acidentes de trabalho(16).

Especificamente, quanto aos índices de estresse, o presente estudo apontou a presença expressiva de participantes com manifestações sintomáticas desse agravo. Pesquisa realizada com profissionais que trabalham na $A B$ em Belo Horizonte/MG, Brasil, mostrou que 19,4\% dos profissionais apresentaram estresse laboral em função da elevada carga e alta exigência do trabalho ${ }^{(17)}$. 
O presente estudo também apontou uma incidência expressiva de sintomas ansiosos entre os participantes. Estudo realizado junto a profissionais que atuavam em Unidade de Terapia Intensiva no Estado de São Paulo, Brasil, detectou sintomas de ansiedade em $15 \%$ dos sujeitos avaliados ${ }^{(18)}$.

Sintomas depressivos também foram verificados junto aos participantes da presente pesquisa. Em estudos realizados com profissionais de equipes de enfermagem que atuavam em contextos hospitalares a prevalência de manifestações sintomáticas de depressão variou de $13 \%$ a $38 \%{ }^{(19-20)}$ e estavam relacionadas às condições inadequadas de trabalho e longas jornadas laborais.

Um ponto muito importante levantado pela presente investigação é que os casos de consumo de bebidas alcoólicas no padrão binge estão claramente associados a sintomas de depressão. Não foram encontrados estudos disponíveis em bases de dados indexadas que tenham avaliado a relação entre o uso de álcool e depressão especificamente em profissionais de enfermagem da ESF.

Pesquisas apontam a existência de uma tendência de comorbidade entre a presença de depressão e o uso abusivo de álcool, de álcool e/ou outras drogas. Esse aspecto merece destaque, uma vez que a presença de depressão e o abuso de substâncias estão entre os principais fatores predisponentes para suicídio entre profissionais de enfermagem ${ }^{(21-22)}$. No presente estudo foram encontradas correlações entre depressão e estresse, depressão e ansiedade, bem como entre estresse e ansiedade. Essas correlações também foram reportadas por outras pesquisas, nas quais o ambiente de trabalho extenuante e insalubre desponta como principal fator desencadeador de estresse concomitantemente com outros sintomas sugestivos de sofrimento psíquico $^{(19,22)}$.

Podem-se observar correlações negativas entre estresse e idade, bem como entre estresse e tempo de trabalho, o que sugere que quanto menos idade e tempo de trabalho dos profissionais de enfermagem, maiores são os níveis de estresse. Esses resultados também foram encontrados em estudos realizados em Taiwan e Austrália ${ }^{(23-24)}$. Uma das possibilidades de explicação para esse fenômeno seria o fato de que, quando mais jovens são as enfermeiras, maiores seriam suas atribuições domésticas, resultando em sobrecarga de trabalho e aumento da pressão por resultados ${ }^{(25)}$.

Uma das limitações que este estudo apresenta se refere ao fato de ter sido realizado com profissionais de enfermagem que atuam em equipes da ESF de um único município. Outro ponto a ser destacado, mas que não se refere especificamente ao delineamento da presente pesquisa, é a escassez de estudos que envolvem a relação entre os sintomas de transtorno mental e o uso de substâncias psicoativas por profissionais de enfermagem que atuam na ESF, o que torna difícil a comparação com resultados de pesquisas realizadas em outros contextos.

\section{CONCLUSÃO}

Este estudo mostrou que os profissionais da enfermagem que atuam em equipes da ESF apresentam níveis consideráveis de consumo de bebidas alcoólicas no padrão binge, e que esse consumo problemático está relacionado a maiores níveis sintomáticos de depressão. Outros resultados relevantes obtidos são que os profissionais do sexo masculino apresentam maior vulnerabilidade ao uso abusivo de álcool e outras drogas e os trabalhadores com menor idade e tempo de atuação tendem a apresentar maiores níveis de estresse. 
Os resultados encontrados podem contribuir para a superação de deficiências reportadas na adoção de estratégias específicas voltadas para a promoção de saúde mental do trabalhador de enfermagem que atua nos cenários da ESF. Esses profissionais se deparam em sua rotina laboral com as particularidades que revestem esse campo de atuação, o que os coloca em contato com uma multiplicidade de desafios em suas práticas cotidianas que podem aumentar sua vulnerabilidade aos transtornos mentais.

\section{REFERÊNCIAS}

1. Ministério da Saúde, Secretaria de Atenção à Saúde, Departamento de Atenção Básica. Política Nacional de Atenção Básica [Internet]. Brasília: Ministério da Saúde; 2012 [acesso em: 03 out. 2018]. Disponível em:

http://189.28.128.100/dab/docs/publicacoes/geral/pnab.pdf.

2. Pinto LF, Rocha CMF, Lapão LV, Pisco LAC. Sistemas Comparados de Saúde: Atenção Primária à Saúde nas cidades de Lisboa e do Rio de Janeiro. Cien Saude Colet [Internet]. 2017 [acesso em: 03 out. 2018];22(3):676-7. Disponível em:

https://doi.org/10.1590/1413-81232017223.00202017.

3. Dilélio AS, Facchini LA, Tomasi E, Silva SM, Thumé E, Piccini RX, et al. Prevalência de transtornos psiquiátricos menores em trabalhadores da atenção primária à saúde das regiões Sul e Nordeste do Brasil. Cad Saude Publica [Internet]. 2012 [acesso em: 03 out. 2018];28(3):503-14. Disponível em: https://doi.org/10.1590/S0102-311X2012000300011.

4. Goulart Junior E, Feijó MR, Cunha EV, Correa BJ, Gouveia PAES. Exigências familiares e do trabalho: um equilíbrio necessário para a saúde de trabalhadores e organizações. Pensando familias [Internet]. 2013 [acesso em: 03 out. 2018];17(1):110-22. Disponível em: http://pepsic.bvsalud.org/scielo.php?script=sci arttext\&pid=S1679-494X2013000100011.

5. Darbro N, Malliarakis KD. Substance Abuse: Risks Factors and Protective Factors. J Nurs Regul [Internet]. 2012 [acesso em: 03 out. 2018];3(1):44-8. Disponível em: https://doi.org/10.1016/\$2155-8256(15)30234-9.

6. Carvalho DB, Araújo TM, Bernardes KO. Transtornos mentais comuns em trabalhadores da Atenção Básica à Saúde. Revista Brasileira de Saúde Ocupacional [Internet]. 2016 [acesso em: 03 out. 2018];41:e17. Disponível em: https://doi.org/10.1590/2317$\underline{6369000115915 .}$

7. Moreira IJB, Horta JA, Duro LN, Borges DT, Cristofari AB, Chaves J, et al. Perfil sociodemográfico, ocupacional e avaliação das condições de saúde mental dos trabalhadores da Estratégia Saúde da Família em um município do Rio Grande do Sul, RS. Rev Bras Med Família e Comunidade [Internet]. 2016 [acesso em: 03 out. 2018];11(38):1-12. Disponível em:

https://doi.org/10.5712/rbmfc11(38)967.

8. Henrique IFS, De Micheli D, Lacerda RB, Lacerda LA, Formigoni MLOS. Validação da versão brasileira do teste de triagem do envolvimento com álcool, cigarro e outras substâncias (ASSIST). Rev Assoc Med Bras (1992) [Internet]. 2004 [acesso em: 03 out. 2018];50(2):199-206. Disponível em: https://doi.org/10.1590/S0104-42302004000200039.

9. Meneses-Gaya C, Zuardi AW, Loureiro SR, Hallak JE, Trzesniak C, Marques JMA, et al. Is the full version of the AUDIT really necessary? Study of the validity and internal construct of its abbreviated versions. Alcohol Clin Exp Res [Internet]. 2010 [acesso em: 03 out. 2018];34(8):1417-24. Disponível em: https://doi.org/10.1111/j.1530-0277.2010.01225.x.

10. Vignola RC, Tucci AM. Adaptation and validation of the depression, anxiety and stress scale (DASS) to Brazilian Portuguese. J Affect Disord [Internet]. 2014 [acesso em: 03 out. 2018];155:104-9. Disponível em: https://doi.org/10.1016/j.jad.2013.10.031. 11. Hidalgo CL, Vargas Casas GM Salcedo Monsalve A. Consumo de sustancias psicoactivas en profesionales de la salud (médicos y enfermeros) de dos IPS de primer nivel de atención en consulta externa de Bogotá. Revista Ciencias de la Salud [Internet]. 2012 [acesso em: 03 out. 2018];10(esp.):87-100. Disponível em: https://revistas.urosario.edu.co/index.php/revsalud/article/view/2030. 12. Kunyk D. Substance use disorders among registered nurses: prevalence, risks and perceptions in a disciplinary jurisdiction. J Nurs Manag [Internet]. 2015 [acesso em: 03 out. 2018];23(1):54-64. Disponível em: https://doi.org/10.1111/jonm.12081.

13. World Health Organization. Global status report on alcohol and health [Internet]. Geneva: World Health Organization; 2011 [acesso em: 03 out. 2018]. Disponível em:

http://www.who.int/entity/substance_abuse/publications/global_alcohol_report/msbgsruprofiles.pdf?ua=1.

14. Oliveira EB, Fabri JMG, Paula GS, Souza SRC, Silveira WG, Matos GS. Padrões de uso de álcool por trabalhadores de enfermagem e a associação com o trabalho. Rev. enferm. UERJ [Internet]. 2013 [acesso em: 03 out. 2018];21(esp. 2):729-35. Disponível em:

http://www.e-publicacoes.uerj.br/index.php/enfermagemueri/article/view/11514.

15. Mahalik JR, Lombardi CM, Sims J, Coley RL, Lynch AD. Gender, male-typicality, and social norms predicting adolescent alcohol intoxication and marijuana use. Soc Sci Med [Internet]. 2015 [acesso em: 03 out. 2018];143:71-80. Disponível em:

https://doi.org/10.1016/j.socscimed.2015.08.013.

16. Epstein PM, Burns C, Conlon HA. Substance abuse among registered nurses. AAOHN J [Internet]. 2010 [acesso em: 03 out. 2018];58(12):513-6. Disponível em: https://doi.org/10.1177/216507991005801203. 
17. Pimenta AM, Assunção AA. Estresse no trabalho e hipertensão arterial em profissionais de enfermagem da rede municipal de saúde de Belo Horizonte, Minas Gerais, Brasil. Rev. bras. saúde ocup. [Internet]. 2016 [acesso em: 03 out. 2018];41:e6. Disponível em: https://doi.org/10.1590/2317-6369000113515.

18. Oliveira MAC, Pereira IC. Atributos essenciais da Atenção Primária e a Estratégia Saúde da Família. Rev Bras Enferm [Internet]. 2013 [acesso em: 03 out. 2018];66(esp.):158-64. Disponível em: https://doi.org/10.1590/S0034-71672013000700020.

19. Perry L, Lamont S, Brunero S, Gallagher R, Duffield C. The mental health of nurses in acute teaching hospital settings: a crosssectional survey. BMC Nurs [Internet]. 2015 [acesso em: 03 out. 2018];14:15. Disponível em: https://doi.org/10.1186/s12912-0150068-8.

20. Gong Y, Han T, Chen W, Dib HH, Yang G, Zhuang R, et al. Prevalence of anxiety and depressive symptoms and related risk factors among physicians in China: a cross-sectional study. PLoS One [Internet]. 2014 [acesso em: 03 out. 2018];9(7):e103242. Disponível em: https://doi.org/10.1371/journal.pone.0103242.

21. Alderson M, Parent-Rocheleau X, Mishara B. Critical Review on Suicide Among Nurses. Crisis [Internet]. 2015 [acesso em: 03 out. 2018];36(2):91-101. Disponível em: https://doi.org/10.1027/0227-5910/a000305.

22. Ferreira LAL, Ferreira LL. Depressão no trabalho da enfermagem: revisão de literatura. Universitas: Ciências da Saúde [Internet]. 2015 [acesso em: 03 out. 2018];13(1):41-8. Disponível em: https://doi.org/10.5102/ucs.v13i1.2849.

23. Chiang YM, Chang Y. Stress, depression, and intention to leave among nurses in different medical units: implications for healthcare management/nursing practice. Health Policy [Internet]. 2012 [acesso em: 03 out. 2018];108(2-3):149-57. Disponível em: https://doi.org/10.1016/j.healthpol.2012.08.027.

24. Hegney DG, Craigie M, Hemsworth D, Osseiran-Moisson R, Aoun S, Francis K, et al. Compassion satisfaction, compassion fatigue, anxiety, depression and stress in registered nurses in Australia: study 1 results. J Nurs Manag [Internet]. 2014 [acesso em: 03 out. 2018];22(4):506-18. Disponível em: https://doi.org/10.1111/jonm.12160.

25. Pereira AV. Nurses' daily life: gender relations from the time spent in hospital. Rev Lat Am Enfermagem [Internet]. 2015 [acesso em: 03 out. 2018];23(5):945-53. Disponível em: https://doi.org/10.1590/0104-1169.0485.2635. 